\title{
Energetics and Defect Clustering Trends for Trivalent Rare Earth Cations Substituted in $\mathrm{UO}_{2}$
}

\author{
J.M. Solomon ${ }^{\mathrm{a}, *}$, A. Navrotsky ${ }^{\mathrm{b}, \mathrm{c}}$, M. Asta ${ }^{\mathrm{a}, \mathrm{c}}$ \\ ${ }^{a}$ Department of Materials Science and Engineering, University of California, Berkeley, \\ CA 94720, USA \\ ${ }^{b}$ Peter A. Rock Thermochemistry Laboratory and NEAT ORU, University of California, \\ Davis, California 95616, USA \\ ${ }^{c}$ Department of Chemical Engineering and Materials Science, University of California, \\ Davis, California 95616, USA
}

\begin{abstract}
Trends in the energetics and defect clustering tendencies for $\mathrm{UO}_{2}$ compounds substituted with trivalent rare earth cations $\left(\mathrm{M}^{3+}\right)$ are investigated computationally using methods based on density functional theory. Higher energetic stability of $\mathrm{U}_{1-x} \mathrm{M}_{x} \mathrm{O}_{2-0.5 x}$ solid solutions relative to constituent oxides and increased preference for higher oxygen coordination around the trivalent cation are found with increasing size of the $\mathrm{M}^{3+}$ species. The implications of the computational results for the effect of trivalent fission products on oxygen ion mobility in spent fuel are discussed.
\end{abstract}

\section{Introduction}

A key concern of the nuclear materials community is the assurance of safe long-term storage of spent nuclear fuel. In this context, an issue that has received considerable attention is the effect that the substitution of $\mathrm{U}^{4+}$ cations by more soluble trivalent rare earth fission product cations $\left(\mathrm{M}^{3+}\right)$ has on the rate of oxidation and oxidative corrosion in uranium oxide $\left(\mathrm{UO}_{2}\right)$ $[1,2,3,4,5,6,7]$. In particular, recent studies $[8,1]$ attributed the trend towards decreased oxidative dissolution rates with increasing burnup, to the interactions between cations and charge-compensating defects (i.e., oxygen vacancies) in the formation of stable defect clusters. The authors in ref.

\footnotetext{
${ }^{*}$ Corresponding author. email: jsolom@berkeley.edu
} 
[1] found evidence of such defect clusters with increasing trivalent rare earth doping level using Raman spectroscopic techniques. These clusters may form by the thermodynamically-favorable association of trivalent substitutional atoms $\left(\mathrm{M}^{3+}\right)$ with charge-compensating oxygen vacancies. The formation of such stable clusters has been hypothesized to reduce the concentration of mobile (unbound) oxygen vacancies, and thus is expected to lead to a lowering of oxygen ion diffusivity $[1,2,8]$. Cluster formation may thus be expected to reduce the rate of oxidation and eventual dissolution of the fuel in repository waste storage containers for both thermodynamic (by stabilizing the solid solution) and kinetic (by reducing oxygen mobility) reasons.

While the effect of rare earth substitution on oxygen chemical potentials, lattice constants and thermal conductivity in $\mathrm{UO}_{2}$ has been well studied (e.g., $[9,10]$ and references therein), and initial experimental and computational studies of formation energetics in urania-rare earth solid solutions have been recently reported $[11,12]$, explicit investigations of defect-clustering behavior has been addressed in relatively few studies $[12,13,14]$. Computational modeling based on classical ionic pair potentials [14] and defect chemistry models [13] concludes that defect clustering is energetically favorable (as it must be in order for clustering to occur, because clustering decreases the entropy); however, a detailed study of systematic trends in energetics and clustering tendencies with varying concentrations and types of rare earth trivalent cations $\left(\mathrm{M}^{3+}\right)$ has not been reported to the best of our knowledge. As a consequence, understanding the effects of defect clustering on solid-solution stability and oxygen vacancy mobilities, which is key in understanding fuel oxidation/corrosion behavior, remains incomplete.

Previous studies $[15,16,17]$ on trivalent-doped fluorite systems concluded that oxygen vacancy mobility is highest when there is little energetic preference for a particular defect ordering arrangement. In a previous computational study by the authors [12] employing density-functionaltheory-based methods, different energetic properties and defect clustering tendencies were obtained for oxygen-vacancy-charge-compensated $\mathrm{UO}_{2}$ systems $\left(\mathrm{U}_{1-x} \mathrm{M}_{x} \mathrm{O}_{2-0.5 x}\right)$ containing the smallest and largest rare earth trivalent cations (M), Y and La, respectively. That work suggested that the magnitude and nature of vacancy interactions with trivalent cations are influenced by the ionic radius of the $\mathrm{M}^{3+}$ species. However, the results were based on calculations for only two rare earth cations, and further work is warranted to better establish and understand such trends. In the present work, the energetics are further investigated by considering a series of trivalent rare earth cations 
substituted in $\mathrm{UO}_{2}$ solid solutions with charge compensating oxygen vacancies. Specifically, we consider systems of composition $\mathrm{U}_{0.5} \mathrm{M}_{0.5} \mathrm{O}_{1.75}$, with $\mathrm{M}$ being $\mathrm{Dy}^{3+}$ (1.03 $\AA$, Shannon radius with eightfold coordination [18]), $\mathrm{Gd}^{3+}$ $(1.05 \AA), \mathrm{Eu}^{3+}(1.07 \AA), \mathrm{Sm}^{3+}(1.08 \AA), \mathrm{Pm}^{3+}(1.09 \AA), \mathrm{Nd}^{3+}(1.11 \AA), \mathrm{Pr}^{3+}$ $(1.13 \AA), \mathrm{Ce}^{3+}(1.14 \AA)$ and $\mathrm{La}^{3+}(1.16 \AA)$. The results reveal clear trends that are discussed in the context of models that have been proposed for other trivalent-doped fluorite-structured oxide systems. We note that in the current calculations we do not consider uranium in oxidation states higher than +4 , so the total vacancy concentration is fixed by the amount of rare earth substitution, with no oxygen interstitials present.

\section{Computational Methodology}

The computational approach employed in this work has been described previously [12], and is summarized briefly in this section. The lowest-energy structure for a given concentration of a particular $\mathrm{M}^{3+}$ species (and corresponding number of oxygen vacancies) is determined by calculation of many hypothetical structures with different configurations of $\mathrm{U}^{4+}$ and $\mathrm{M}^{3+}$ on the cation sublattice, and oxygen ions and vacancies on the anion sublattice of the fluorite structure. This involves the use of ionic pair potential models $[19,20,15,21,22]$ to screen out configurations with large energies, and the use of DFT-based methods to determine energies of the lowest-energy structures. The DFT energies were obtained within the formalism of the projector augmented-wave (PAW) method $[23,24]$ and the Perdew-Burke-Ernzerhof (PBE) generalized gradient approximation (GGA) $[25,26]$ as implemented in the Vienna ab initio simulation package (VASP) [27, 28]. A Hubbard-U correction in the DFT $+\mathrm{U}$ formalism of Dudarev et al. [29] with $\mathrm{U}_{\text {eff }}=\mathrm{U}-\mathrm{J}$ $=4.00 \mathrm{eV}$ was employed for the treatment of the uranium $5 f$ orbitals in this study. We employ the approach by Meredig et al. [30] involving a gradual ramping up of the Hubbard-U parameter. In our previous work [12] we compared this ramping method to the Occupation Matrix Control (OMC) method by Dorado et al. [31], in which we sampled 61 different occupation matrices for a trivalent doped fluorite $\mathrm{UO}_{2}$ system charged-compensated with oxygen vacancies, and found that the ramping method gave rise to a configuration with a lower total energy than that derived using OMC. The PAW potentials used in this work are U, O, Dy_3, Gd_3, Eu_3, Sm_3, Pm_3, Nd_3, Pr_3, and Ce_3 in the VASP PBE library, where the "_3" denotes that the occupied $4 f$ orbitals in the trivalent oxidation state of the rare-earth ions are 
treated as core electrons. We employ a plane-wave cutoff energy of $500 \mathrm{eV}$, and the Brillouin zone is sampled using the Monkhorst-Pack scheme with 4 $\mathrm{x} 4 \mathrm{x} 4 \mathrm{k}$-point meshes. Atomic positions were relaxed with no symmetry constraints until residual forces were below approximately $20 \mathrm{meV} / \AA$. From convergence checks with respect to plane-wave cutoff and k-point sampling we estimate the formation enthalpies are converged at the level of a few tenths of a $\mathrm{kJ} / \mathrm{mol}$-cation.

The formation enthalpy for the $\mathrm{U}_{0.5} \mathrm{M}_{0.5} \mathrm{O}_{1.75}$ compounds considered here, with respect to its constituent oxides, is defined as

$$
\Delta H_{f}=E\left(U_{0.5} M_{0.5} O_{1.75}\right)-\left[E\left(U O_{2}\right)-E\left(M O_{1.5}\right)\right] / 2,
$$

where the energies $(E)$ are expressed per cation. In Eq. 1, $E\left(U O_{2}\right)$ is for fluorite-structured $\mathrm{UO}_{2}$ (calculated here using a 12-atom unit cell with $1 \mathrm{k}$ antiferromagnetic order), and $E\left(M O_{1.5}\right)$ is for $\mathrm{M}_{2} \mathrm{O}_{3}$ in the experimentally observed (i.e., room temperature) cubic bixbyite (C-type sesquioxide) or hexagonal (A-type) structure. The experimentally observed C-type sesquioxides are $\mathrm{Y}_{2} \mathrm{O}_{3}, \mathrm{Dy}_{2} \mathrm{O}_{3}, \mathrm{Gd}_{2} \mathrm{O}_{3}, \mathrm{Eu}_{2} \mathrm{O}_{3}, \mathrm{Sm}_{2} \mathrm{O}_{3}$, and $\mathrm{Pm}_{2} \mathrm{O}_{3}$, and the A-type sesquioxides are $\mathrm{Nd}_{2} \mathrm{O}_{3}, \mathrm{Pr}_{2} \mathrm{O}_{3}, \mathrm{Ce}_{2} \mathrm{O}_{3}$, and $\mathrm{La}_{2} \mathrm{O}_{3}$ [32].

In this study we investigated $\mathrm{M}^{3+}$ substitution levels that are high relative to typical fission product yields. This was done for several reasons. It allows for the practical application of high throughput calculations for systems with various dopant species. It also allows for direct comparison with previous results that considered $\mathrm{M}^{3+}=\mathrm{Y}^{3+}$ and $\mathrm{La}^{3+}$, which were compared to other fluorite oxide systems where high $\mathrm{M}^{3+}$ substitution levels are common [12].

\section{Results and Discussion}

We begin by analyzing the calculated formation enthalpies for the lowestenergy structures of $\mathrm{U}_{0.5} \mathrm{M}_{0.5} \mathrm{O}_{1.75}$ plotted as a function of the Shannon ionic radius of the $\mathrm{M}^{3+}$ cation for eightfold coordination (Fig. 1). Positive values of $\Delta H_{f}$ indicate that phase separation into $\mathrm{UO}_{2}$ and $\mathrm{M}_{2} \mathrm{O}_{3}$ is favored (endothermic) at low temperatures, whereas negative values of $\Delta H_{f}$ indicate a compound forming tendency (exothermic). Figure 1 illustrates that compound formation is preferred (weakly) only for the largest $\mathrm{M}^{3+}$ cation sizes, while the formation enthalpy is increasingly positive as the $\mathrm{M}^{3+}$ ionic radius decreases.

The magnitudes of the formation enthalpies are relatively small in comparison to the magnitude of the configurational entropy contribution to the 


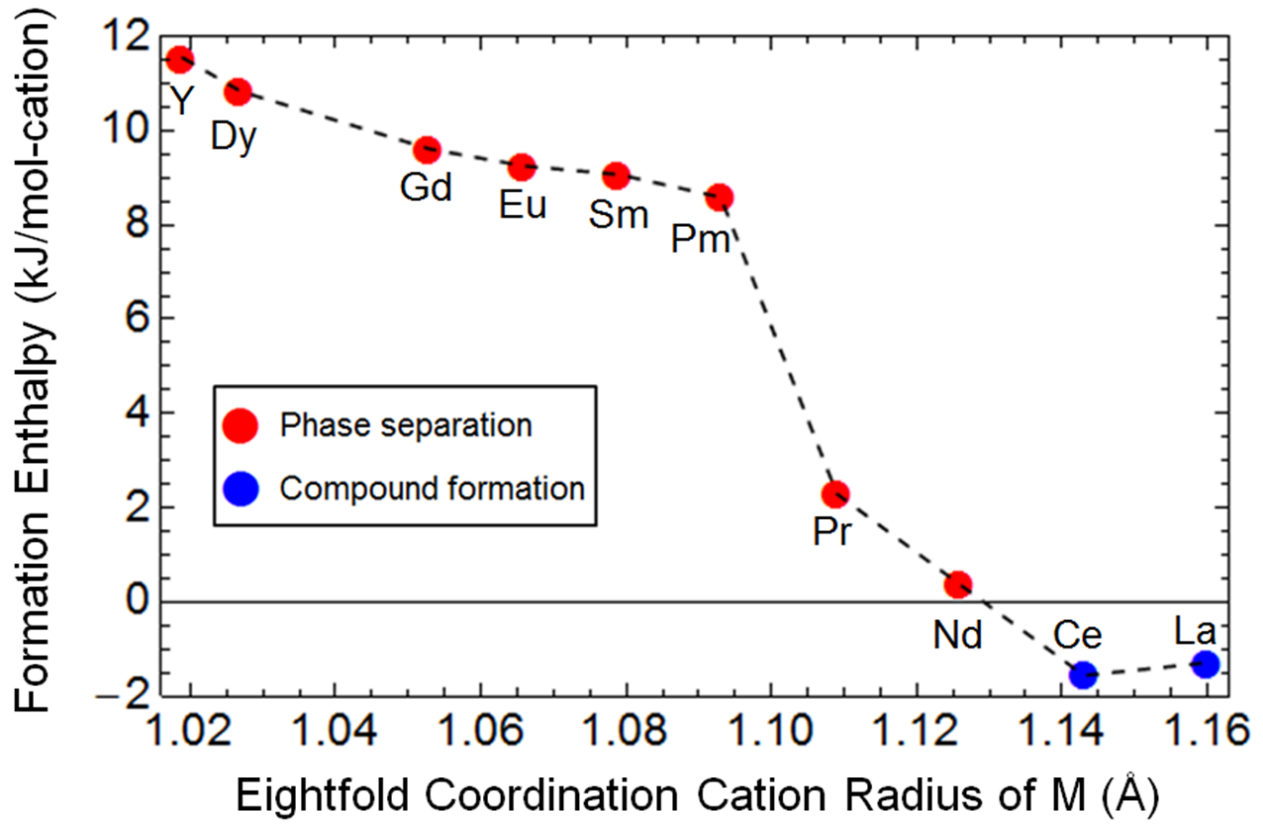

Figure 1: Formation enthalpies of $\mathrm{U}_{0.5} \mathrm{M}_{0.5} \mathrm{O}_{1.75}$ structures are shown for the lowestenergy fully relaxed structures of all compositions enumerated in this study. Calculations for $\mathrm{M}=\mathrm{Y}$ and La were taken from ref. [12] for comparison.

Gibbs free energy at temperatures relevant to the processing and service of nuclear fuels. For example, for a $\mathrm{U}_{1-x} \mathrm{M}_{x} \mathrm{O}_{2-0.5 x}$ system with $x=0.5$ the ideal configurational entropy leads to a contribution of $-12 \mathrm{~kJ} /$ mol-cation to the Gibbs free energy of formation at $\mathrm{T}=1000 \mathrm{~K}$, which is larger than the magnitude of $\Delta H_{f}$ for all the trivalent species plotted in Fig. 1. Consequently the formation free energy is expected to be negative at such temperatures for all of the systems considered in this work. This result is consistent with the fact that rare-earth fission products typically show solubility in $\mathrm{UO}_{2}$ over wide composition and temperature ranges [33].

We consider now the defect-clustering tendencies in $\mathrm{UO}_{2}-\mathrm{M}_{2} \mathrm{O}_{1.5}$ systems by examining the nature of preferred interactions between $\mathrm{M}^{3+}$ and oxygen vacancies. The formation enthalpies of three low energy structures with one, two, and three $\mathrm{M}^{3+}$-oxygen vacancy nearest neighbors averaged over all $\mathrm{M}^{3+}$ ions in the system (two total) are compared for each $\mathrm{M}^{3+}$ species in Fig. 2 , denoted by the blue, green, and red circles, respectively. The structural motifs of the three structures are represented in Fig. 3. 


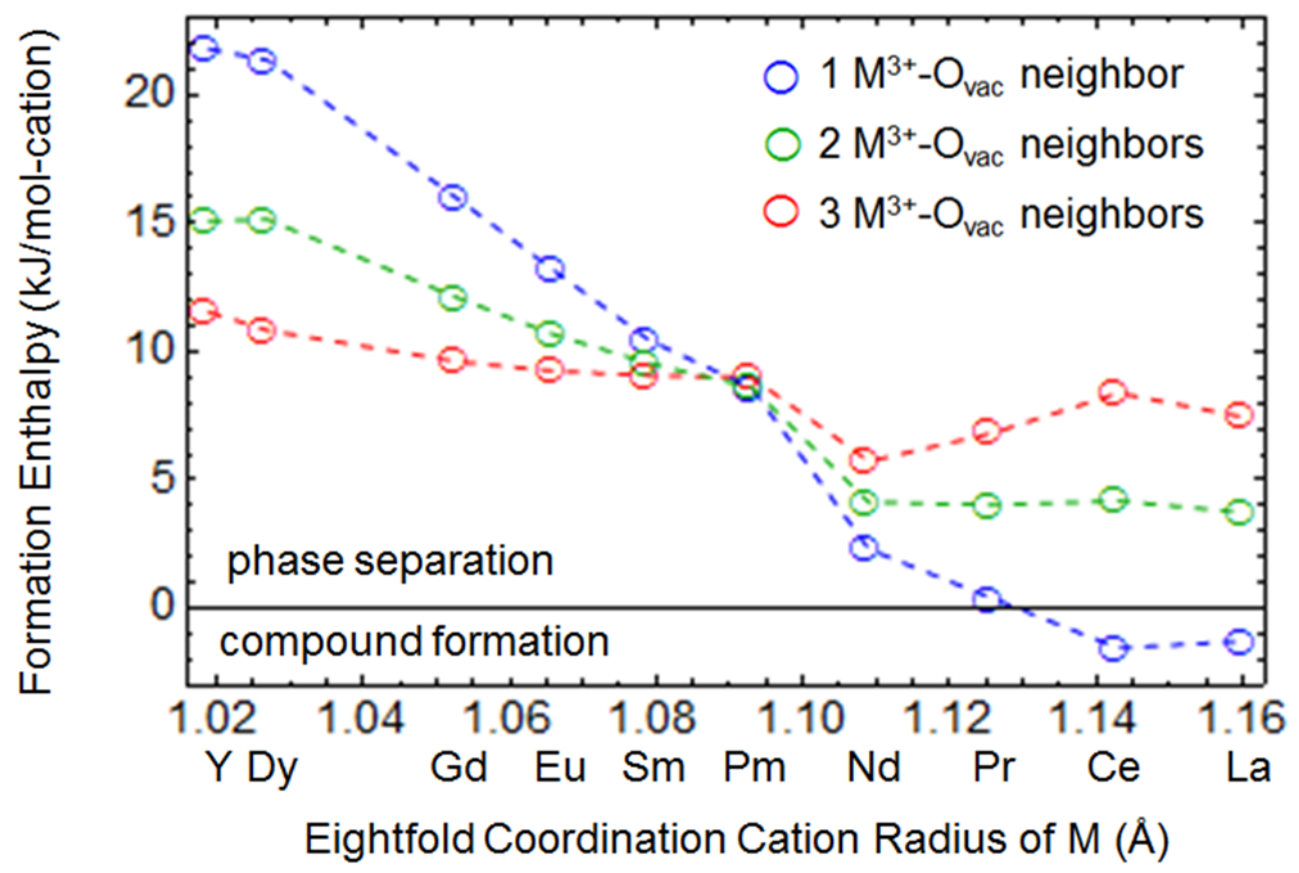

Figure 2: The formation enthalpies for selected structures containing, one, two, and three trivalent cation nearest neighbors to an oxygen vacancy are shown. Calculations for $\mathrm{M}=\mathrm{Y}$ and La were taken from ref. [12] for comparison.

The results in Fig. 2 demonstrate that the small and large $\mathrm{M}$ cations display qualitatively different preferences; the formation enthalpies of systems containing smaller and larger $\mathrm{M}$ cations decrease (more exothermic) and increase (more endothermic), respectively, with increasing number of $\mathrm{M}^{3+}$-vacancy neighbors. For an $\mathrm{M}$ cation size of $1.09 \AA(\mathrm{M}=\mathrm{Pm})$, the formation enthalpies for the three configurations are nearly degenerate, i.e. there is little energetic preference for $\mathrm{M}^{3+}$-oxygen vacancy and $\mathrm{U}^{4+}$-oxygen vacancy nearest neighbors. Similar observations of a trivalent cation size for which there is an apparent lack of preferred clustering tendencies has been found in previous calculations for $\mathrm{CeO}_{2}[15,34]$ and $\mathrm{ZrO}_{2}[16,35]$ fluorite structures.

The trends in Figs. 1 and 2 are consistent with models presented in the literature to explain similar results in other fluorite-structured oxide systems $[36,37]$. We first consider the trend of lower formation enthalpy (more exothermic) with increasing size of the $\mathrm{M}^{3+}$ rare earth ion in Fig. 1. $\mathrm{U}^{4+}$ prefers an eightfold oxygen ion coordination environment. While of similar 

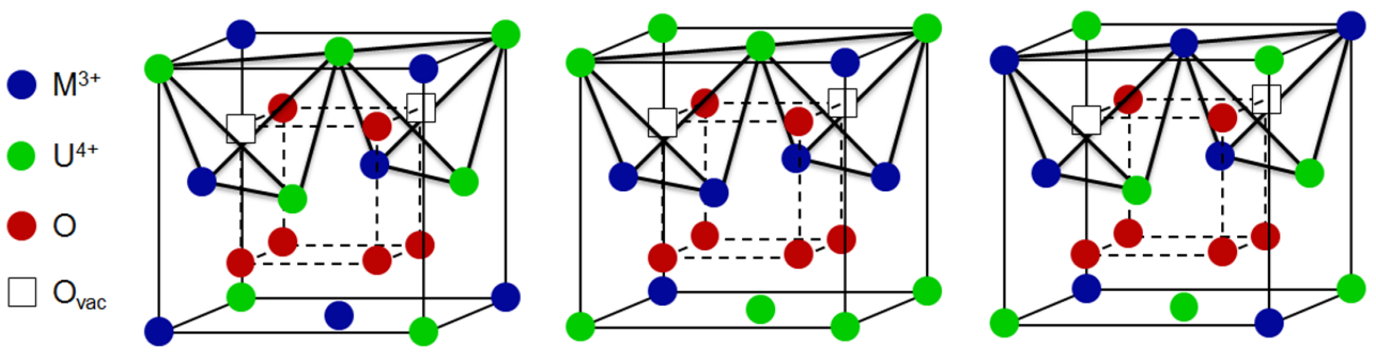

Figure 3: Structural motifs of low energy structures containing one (left), two (middle), and three (right) $\mathrm{M}^{3+}$-oxygen vacancy nearest neighbors.

size to $\mathrm{U}^{4+}$, the rare-earth $\mathrm{M}^{3+}$ cations considered in this work generally prefer a lower coordination environment due to their lower valence. Upon formation of the defected fluorite compound $\mathrm{U}_{1-x} \mathrm{M}_{x} \mathrm{O}_{2-0.5 x}, \mathrm{U}^{4+}$ in general must lower its coordination environment relative to its coordination in pure $\mathrm{UO}_{2}$ due to the formation of charge compensating oxygen vacancies; concomitantly, $\mathrm{M}^{3+}$ must increase its coordination environment relative to pure $\mathrm{M}_{2} \mathrm{O}_{3}$. Increasing energetic stability of the $\mathrm{U}_{1-x} \mathrm{M}_{x} \mathrm{O}_{2-0.5 x}$ compounds with increasing size of the $\mathrm{M}^{3+}$ cation can then be understood as resulting from the lower energetic penalty associated with increasing the oxygen coordination around larger rare earth ions.

The trend in Fig. 2 shows that the preference for oxygen vacancy nearest neighbors decreases with increasing size of the $\mathrm{M}^{3+}$ cations, which is due to larger ions preferring higher oxygen ion coordination. The preference for a particular defect ordering arrangement, i.e. for rare earth cations with size smaller or larger than the radius at the crossover point, is suggestive of defect clustering in short range ordered solid solutions. This clustering effect is expected to lower oxygen ion mobility, as discussed previously in the context of ionic conductivity in fluorite-structured solid electrolytes [38], by limiting the number of oxygen vacancies available for oxygen to diffuse into the solid. This kinetic limitation of oxygen incorporation due to clustering is believed to reduce oxidation and subsequent dissolution of spent fuel $[8,1]$. The work in ref. [1] which found evidence of stable defect clusters and reduced fuel dissolution with increasing $\mathrm{M}=\mathrm{Gd}$ and Dy dopants is consistent with our calculations which show strong preference for ordering/clustering for these systems (Fig. 2), supporting the hypothesis that clustering is present and that it kinetically limits fuel corrosion. 


\section{Summary}

A systematic DFT study of $\mathrm{UO}_{2}$ compounds substituted with trivalent rare earth cations $\left(\mathrm{M}^{3+}\right)$ found higher energetic stability of $\mathrm{U}_{1-x} \mathrm{M}_{x} \mathrm{O}_{2-0.5 x}$ solid solutions relative to constituent oxides with increasing size of the $\mathrm{M}^{3+}$ species. Furthermore, an investigation of the defect ordering tendencies shows an increasing preference for higher oxygen coordination around the rare earth ions with increasing size of these $\mathrm{M}^{3+}$ species. Compounds with ionic radius of the $\mathrm{M}^{3+}$ species smaller or larger than $1.09 \AA$ are found to have energetically preferred defect ordering arrangements. Systems with preferred defect ordering arrangements are suggestive of defect clustering in short range ordered solid solutions, which is expected to limit oxygen ion mobility and therefore the rate of oxidation of spent nuclear fuel.

\section{Acknowledgments}

J.M.S. was supported by the Office of Basic Energy Sciences of the U.S. Department of Energy as part of the Materials Science of Actinides Energy Frontier Research Center (DE-SC0001089) for initial DFT+U calculations, and the Department of Defense through the National Defense Science \& Engineering Graduate Fellowship Program for the remainder of the work. A.N. and M.A. were supported by the U.S. Department of Energy as part of the Materials Science of Actinides Energy Frontier Research Center (DESC0001089). This work made use of resources of the National Energy Research Scientific Computing Center, supported by the Office of Basic Energy Sciences of the U.S. Department of Energy under Contract No. DE-AC02$05 \mathrm{CH} 11231$.

[1] M. Razdan, D. W. Shoesmith, Journal of The Electrochemical Society 161 (2014) H105-H113.

[2] M. Razdan, D. W. Shoesmith, Journal of The Electrochemical Society 161 (2014) H225-H234.

[3] R. McEachern, P. Taylor, Journal of Nuclear Materials 254 (1998) 87 121.

[4] J.-G. Kim, Y.-K. Ha, S.-D. Park, K.-Y. Jee, W.-H. Kim, Journal of Nuclear Materials 297 (2001) $327-331$. 
[5] L. Thomas, R. Einziger, H. Buchanan, Journal of Nuclear Materials 201 (1993) $310-319$.

[6] R. McEachern, D. Doern, D. Wood, Journal of Nuclear Materials 252 (1998) $145-149$.

[7] E. Aitken, Journal of Nuclear Materials 19 (1966) 248 - 266.

[8] H. He, P. G. Keech, M. E. Broczkowski, J. J. Nol, D. W. Shoesmith, Canadian Journal of Chemistry 85 (2007) 702-713.

[9] D. Shin, T. M. Besmann, Journal of Nuclear Materials 433 (2013) 227 - 232. And references therein.

[10] S. Fukushima, T. Ohmichi, A. Maeda, H. Watanabe, Journal of Nuclear Materials 102 (1981) $30-39$.

[11] L. Mazeina, A. Navrotsky, M. Greenblatt, Journal of Nuclear Materials 373 (2008) $39-43$.

[12] J. Solomon, V. Alexandrov, B. Sadigh, A. Navrotsky, M. Asta, Acta Materialia 78 (2014) $282-289$.

[13] K. Park, D. Olander, Journal of Nuclear Materials 187 (1992) 89 - 96.

[14] S. Middleburgh, D. Parfitt, R. Grimes, B. Dorado, M. Bertolus, P. Blair, L. Hallstadius, K. Backman, Journal of Nuclear Materials 420 (2012) 258 $-261$.

[15] L. Minervini, M. O. Zacate, R. W. Grimes, Solid State Ionics 116 (1999) $339-349$.

[16] A. Bogicevic, C. Wolverton, G. M. Crosbie, E. B. Stechel, Phys. Rev. B 64 (2001) 014106.

[17] A. Bogicevic, C. Wolverton, Phys. Rev. B 67 (2003) 024106.

[18] R. D. Shannon, Acta Crystallographica Section A 32 (1976) 751-767.

[19] R. W. Grimes, D. J. Binks, A. B. Lidiard, Philosophical Magazine A 72 (1995) 651-668. 
[20] L. Minervini, R. W. Grimes, K. E. Sickafus, Journal of the American Ceramic Society 83 (2000) 1873-1878.

[21] G. Busker, A. Chroneos, R. W. Grimes, I.-W. Chen, Journal of the American Ceramic Society 82 (1999) 1553-1559.

[22] C. R. Stanek, L. Minervini, R. W. Grimes, Journal of the American Ceramic Society 85 (2002) 2792-2798.

[23] P. E. Blöchl, Phys. Rev. B 50 (1994) 17953-17979.

[24] G. Kresse, D. Joubert, Phys. Rev. B 59 (1999) 1758-1775.

[25] J. P. Perdew, K. Burke, M. Ernzerhof, Phys. Rev. Lett. 77 (1996) 38653868.

[26] J. P. Perdew, K. Burke, M. Ernzerhof, Phys. Rev. Lett. 78 (1997) 13961396.

[27] G. Kresse, J. Furthmüller, Phys. Rev. B 54 (1996) 11169-11186.

[28] G. Kresse, J. Furthmller, Computational Materials Science 6 (1996) 15 -50 .

[29] S. L. Dudarev, G. A. Botton, S. Y. Savrasov, C. J. Humphreys, A. P. Sutton, Phys. Rev. B 57 (1998) 1505-1509.

[30] B. Meredig, A. Thompson, H. A. Hansen, C. Wolverton, A. van de Walle, Phys. Rev. B 82 (2010) 195128.

[31] B. Dorado, B. Amadon, M. Freyss, M. Bertolus, Phys. Rev. B 79 (2009) 235125.

[32] M. Zinkevich, Progress in Materials Science 52 (2007) 597 - 647.

[33] H. Kleykamp, Journal of Nuclear Materials 131 (1985) 221 - 246. And references therein.

[34] D. A. Andersson, S. I. Simak, N. V. Skorodumova, I. A. Abrikosov, B. Johansson, Proceedings of the National Academy of Sciences of the United States of America 103 (2006) 3518-3521. 
[35] M. O. Zacate, L. Minervini, D. J. Bradfield, R. W. Grimes, K. E. Sickafus, Solid State Ionics 128 (2000) $243-254$.

[36] W. Chen, A. Navrotsky, Journal of Materials Research 21 (2006) 32423251.

[37] M. Aizenshtein, T. Y. Shvareva, A. Navrotsky, Journal of the American Ceramic Society 93 (2010) 4142-4147.

[38] H. Inaba, H. Tagawa, Solid State Ionics 83 (1996) 1 - 16. 